\title{
Abordajes de la salud mental en Atención Primaria de la Salud. Aportes desde una práctica de apoyo matricial ${ }^{1}$
}

\section{Approaches to Mental Health in Primary Health Care. Contributions from a Practice of Matrices Support}

\author{
Florencia Orpinell ${ }^{2}$
}

\section{Resumen}

Este artículo se propone realizar una lectura respecto a las prácticas matriciales de salud mental en al ámbito de la atención primaria de la salud en la ciudad de Rosario, recuperando información obtenida en una investigación realizada en el período 2016-2017.

Partiendo del análisis y descripción de un dispositivo puntual que realiza este tipo de abordajes nos propusimos conocer acerca del apoyo matricial en el campo de la salud mental en nuestra ciudad y analizar cómo lo matricial nos permite enlazar los abordajes en salud mental de la atención primaria de la salud con diferentes instituciones y organizaciones, fortaleciendo las redes de salud y sus necesarias articulaciones intersectoriales.

Se hallaron diversos modos organizacionales de los equipos que componen el dispositivo, referido- por sus protagonistas - a diseños poco claros en la planificación de este dispositivo como parte de estrategias de políticas en Salud Mental.
Sin embargo, en la heterogeneidad de las prácticas registradas se pudieron hallar puntos de encuentro: 1) Incidir en los modelos de atención institucional promoviendo espacios interdisciplinarios de revisión de estrategias de atención con los equipos de los centros de salud, 2) pensar y promover otras posibilidades de alojar las problemáticas en salud mental abriendo espacios hacia y con la comunidad, 3) la articulación intersectorial y, 4) interrogar las diferencias entre un modelo clínico asistencialista que solo atiende al síntoma desde dispositivos convencionales, reduciendo al sujeto a una práctica biomédica y la atención comunitaria que toma la demanda específica como oportunidad para realizar una lectura poblacional y crear estrategias de abordaje territorial y colectiva apostando a la clínica de la subjetividad y a la salud mental colectiva desde una perspectiva en derechos humanos.

Palabras claves: Apoyo matricial - Atención Primaria de la salud - Salud comunitaria Sustitución de lógicas manicomiales

\footnotetext{
${ }^{1}$ El presente artículo fue reescrito sobre la base del Trabajo Final Integrador de la Carrera de Especialización en Psicología Clínica, Institucional y Comunitaria "Alcances, límites y desafíos de la implementación de la práctica matricial de Salud Mental en el ámbito de la Atención Primaria de la Salud, en la ciudad de Rosario" del que soy autora y que fue dirigido por la Ps. Ana Cecilia Augsburger.

${ }^{2}$ Especialista en Psicología Clínica, Institucional y Comunitaria. Psicóloga del Dispositivo Territorial de Salud Mental zona sudeste de Rosario. Dirección Provincial de Salud Mental. Ministerio de Salud. Provincia de Santa Fe. Militante en DDHH y Salud Mental. florenciaorpinell@gmail.com
} 


\section{Summary}

This article proposes a reading regarding the matrices practices of mental health in the field of primary health care in the city of Rosario. We have recuperated information obtained in a research carried out in the period 2016-2017. Based on the analysis and description of a specific device that performs this type of approaches we try to know about the matrix support in the field of mental health in our city and to analyze how it could allow us to link the mental health approaches of the primary health care with different institutions and organizations, strengthening health networks and their necessary intersectoral linkages. Various organizational modes of the teams that make up the device were found referred -as their protagonists tell us-, to unclear designs in the planning of this device as part of policy strategies in Mental Health. However, in the heterogeneity of the registered practices, it was possible to find meeting points: 1) impact on institutional care models by promoting interdisciplinary spaces for the review of care strategies with the teams of health centres, 2) think about and promote other possibilities to accommodate mental health problems by opening spaces to and with the community, 3) intersectoral coordination and, 4) question the differences between a clinical care model that only deals with the symptom from conventional devices, -reducing the subject to a biomedical practice-, and the community care which, on the contrary, considers the specific demand as an opportunity to carry out a population reading which can create strategies of territorial and collective approach betting on the clinic of subjectivity and collective mental health from a human rights perspective.

Keywords: Matrices practices - Primary Health Care - Mental Health - Organizational modes

\section{Introducción}

A partir de este escrito nos proponemos indagar sobre la práctica de apoyo matricial en salud mental que realiza un dispositivo matricial dependiente de la Dirección Provincial de Salud Mental (DPSM) en los centros de salud de la ciudad de Rosario, provincia de Santa Fe.

¿Qué es el apoyo matricial? ¿De qué manera se implementa en la ciudad de Rosario? ¿Cuál es la formación que disponen las trabajadoras y los trabajadores de los dispositivos respecto a esta práctica? ¿Cuál es su grado de inclusión dentro de la red de salud? Estos son algunos de los interrogantes que nos han permitido realizar esta investigación en la que se interpretó lo matricial, desde las concepciones elaboradas por las experiencias de la reforma psiquiátrica brasilera; y tomando como referencia las elaboraciones teóricas de Gastón Sousa Campos (2001).

Para definir el apoyo matricial fueron esclarecedores los aportes del artículo Apoio matricial: um estudo sobre a perspectiva de profissionais da saúde mental donde se lo ubica como "un arreglo organizacional que tiene por objetivo proveer soporte técnico especializado a equipos de salud de la atención básica” (Ballarin et al., 2012, p. 768). Desde esta perspectiva y retomando reflexiones de Gastón Sousa Campo (2001), ubicamos que esta modalidad se propone romper con el modelo de asistencia centrado en el médico, como única herramienta de evaluación diagnóstica, incluyendo la construcción interdisciplinaria y la conformación de equipos de referencia. De esta forma, el apoyo matricial, según estos autores:

estimula la producción de espacios que promuevan, en el interior de las U.B.S (Unidades Básicas de Salud), discusiones y prácticas clínicas que tracen nuevos sentidos para las intervenciones en salud, actualizando modos de cuidar que rompan con la lógica de deriva- 
ción a especialistas, o sea, crear otras posibilidades de atención, más allá de los CAPS y hospitales, diversificando y ampliando las alternativas de acogimiento a las necesidades de usuarios con trastornos mentales (Pena, 2009) (Ballarin et al., 2012, p. 768)

El apoyo matricial es entendido, entonces, como un modelo organizacional que se conforma en equipos dispuestos a complementarse con los equipos de referencia de los centros de salud para trabajar determinados casos o situaciones problemáticas de la población.

El apoyador matricial es un especialista (...) que puede agregar recursos de saber y aún contribuir con intervenciones que aumenten la capacidad de resolver problemas de salud del equipo primariamente responsable por el caso. El apoyo matricial busca construir y activar espacios para la comunicación activa y para la circulación de conocimientos entre profesionales de referencia y apoyadores (Campos, Domitti, 2006, p.1549)

Consideramos de gran relevancia poder abordar y sistematizar esta problemática ya que existen escasas producciones escritas en torno a las prácticas de salud mental en la Atención Primaria de la Salud de dependencia provincial en la ciudad de Rosario. Creemos, además, que quienes trabajamos en la salud pública necesitamos producir registros e investigaciones que den cuentan de nuestras prácticas porque es, quizás, a través de estas elaboraciones que vamos capitalizando las experiencias del campo y, a su vez, vamos comprendiendo mediante la escritura, nuestro propio hacer. Hacer que muchas veces se encuentra interpelado o desorientado cuando se trata de aportar a una salud colectiva, comunitaria, que no se agota en la asistencia directa dentro de un consultorio.

Por otro lado, en el contexto actual se hace necesario pensar y construir nuevas alternativas a la lógica manicomial y medicalizante de tratar con el sufrimiento psíquico cuando emerge y nuevos abordajes para acompañar la locura de la vida cotidiana, impidiendo así que no caiga bajo las lógicas de la segregación, discriminación y encierro.

Ante la Ley Nacional de Salud Mental y Adicciones $N^{\circ} 26657$ basada en un paradigma de Derechos Humanos, que interpela la supuesta idea de normalidad, y transforma radicalmente los modos de abordaje y cuidado; ante plazos casi vencidos para lograr la sustitución y cierre de las instituciones manicomiales que, en nombre de la salud, vulneran todo tipo de derechos; ante las demoradas aperturas de redes y servicios alternativos que no logran implementarse es impostergable que las y los protagonistas del campo de la salud mental aportemos estrategias y propuestas para lograr una salud mental integral y colectiva, basada en el respeto, la inclusión y el cuidado.

El enfoque metodológico que se implementó en este trabajo se orientó hacia la construcción de un estudio evaluativo; se pesquisaron las valoraciones respecto al análisis de estructuras, procesos, resultados, así como también la participación y visión de los sujetos involucrados en la creación, organización e implementación de intervenciones sanitarias; incluyendo el análisis de contexto y el contenido de las políticas de salud mental. Con el fin de conocer las evaluaciones mencionadas, se adoptó una perspectiva cualitativa permitiendo una mejor comprensión e interpretación de las diversas miradas entrecruzadas que expresan las entrevistadas y los entrevistados. Mediante la realización de entrevistas abiertas a agentes clave del dispositivo investigado, personal directivo de la DPSM; entrevistas grupales a equipos de centros de salud; análisis de documentos y 
registros de campo se ha logrado elaborar algunos avances en torno al esclarecimiento de estas prácticas, describir sus diferentes modalidades, así como también obtener las valoraciones que le otorgan a la práctica matricial sus protagonistas y los aciertos o dificultades de su implementación.

\section{Atención Primaria de la Salud y Salud Mental. Encuentros y desencuentros.}

Las tensiones y escisiones de estrategias entre quienes planifican las políticas para salud en general y quienes planifican las políticas de salud mental, así como la desarticulación con otros sectores ministeriales han sido y siguen siendo problemáticas cuyos efectos se hacen sentir tanto en la población usuaria de estos servicios como en los relatos de las trabajadoras y los trabajadores o personal directivo a quienes hemos entrevistado.

El primer nivel de atención desde la estrategia de APS, por ser muchas veces el primer lugar donde se reciben problemáticas sociales y subjetivas, ocupa un lugar esencial de contención, elaboración de estrategias y articulación con otras instituciones. Sin embargo, al decir de las trabajadoras entrevistadas y los trabajadores entrevistados cuentan con escasas herramientas para abordar las complejidades de las demandas y para articular con otros servicios que complementen y enriquezcan la atención y respuesta. Así, las derivaciones desde y hacia hospitales generales, las externaciones de monovalentes, las articulaciones con entidades que abordan problemáticas de las infancias o seguridad social como trabajo, vivienda, cultura, discapacidad, sistema judicial, el lazo con dispositivos sustitutivos o con instituciones que alojen situaciones de consumo problemático, se realizan con mucha dificultad. Articulaciones institucionales sostenidas por redes artesanales que las trabajadoras y los trabajadores generan, ligado más bien a lazos de afectividad que a sistemas formales para la referencia y contrareferencia o derivación entre instituciones y servicios.

En nuestra región, la atención primaria para la salud (APS) es una herramienta sanitaria que se desplegó con mucha fuerza hace 30 años, cuando el municipio decidió crear la Dirección de Atención Primaria, como política para el desarrollo del Sistema de Salud de la ciudad; política que, con diferentes características, se venía ya desarrollando en la provincia de Santa Fe. Si bien ha tenido avances y retrocesos según cada gestión de gobierno, se puede destacar que en cada barrio de la ciudad conviven, con más o menos articulación entre sí, centros de salud tanto de dependencia municipal como de dependencia provincial; ofreciendo un sistema de atención de cercanía y gran alcance para la población usuaria del sistema público de salud.

Para el momento de la investigación, la atención primaria de la salud a nivel provincial estaba integrada por 34 centros de salud distribuidos en seis distritos de la ciudad de Rosario. La estructura edilicia y la conformación administrativa de los equipos de cada centro eran muy diversas entre ellas. Edificios nuevos y amplios convivían con viejas casas alquiladas desde la época en que comenzaban a conformarse como vecinales o salitas de primeros auxilios. Sin embargo, en su mayoría se contaba con equipos interdisciplinarios, compuestos por administrativas, administrativos, enfermeras, enfermeros, agentes sanitarios, personal de limpieza, diferentes especialidades médicas y disciplinas como pediatría, ginecología, medicina general, psicología y trabajo social.

Comprendemos la atención primaria de la salud como:

el eslabón inicial de la cadena de atención, donde se resuelven los problemas de menor dificultad técnica -diagnóstica y terapéutica- y se orientan 
los restantes a los niveles sucesivos de la cadena. Ello implica una red de establecimientos interconectados por claros procedimientos de referencia y transmisión de la información pertinente que ordenan la circulación interna de los pacientes en el sistema; también implica un ordenamiento territorial regionalizado y un comportamiento social que sigue, más o menos disciplinadamente, las normas de ingreso y circulación. El tipo de atención que se presta en esos establecimientos no implica una disminución de la calidad de la atención, por el contrario, debe tener la calidad necesaria para poder realizar la distribución señalada; de lo contrario, puede aumentar el riesgo de errores diagnósticos y terapéuticas extemporáneas. (Testa, 2009, p.162)

Sin embargo, Stolkiner (2011) muestra cómo APS quedó afectada por las políticas económicas desiguales que la desproveyó de recursos y le impidió cumplir sus objetivos.

En el marco de la crisis y de la deslegitimación de los discursos de reforma promercado de finales del siglo $\mathrm{X}$

$\mathrm{X}$, se revitalizó la estrategia de Atención Primaria de la Salud. Si bien nunca dejó de estar en los enunciados de las políticas gubernamentales, su implementación en los países de América Latina había adquirido formas que la distanciaron de sus objetivos originales. Dentro de sus distintas interpretaciones posibles, tendió a privilegiarse la APS selectivas reduciéndolas a programas focalizados (Stolkiner, 2011, p. 2808)

Estas políticas atentaban contra la accesibilidad (Augsburger et al.,2012) de la salud que es uno de los objetivos más importantes para abordar la salud en general y, sobre todo, para profundizar un proceso de inclusión de las problemáticas en salud mental.

Ante estas barreras de acceso a la salud mental de la población y ante los escasos recursos para la atención y continuidad del cuidado de personas en sufrimiento psíquico fue que años después de la creación de la Dirección Provincial de Salud Mental en 2005, se crearon dos dispositivos matriciales para realizar una apoyatura a la demanda proveniente de los centros de salud de la ciudad: uno para las infancias y otro para población adulta.

Si bien había diferencias entre ellos, ambos se habían diseñado con el objetivo de cumplimentar la Ley Provincial de Salud Mental $N^{o} 10772$, años previos a la sanción de la Ley Nacional $N^{\circ} 26656$ y con la intensión de fortalecer la salud mental dentro de la atención primaria de la salud; reafirmando la lógica matricial como modalidad de trabajo. La figura de operadores comunitarios y talleristas era característica distintiva de estos dispositivos que ofrecían nuevas modalidades de acercamiento con las usuarias, usuarios y familiares de la salud mental.

En el año 2012 con nuevas autoridades en el Ministerio de Salud y en la Dirección Provincial de Salud Mental, desde el Nodo Rosario (dependencia del Ministerio de salud en la ciudad de Rosario) se propuso hacer una fusión entre los dos dispositivos matriciales aludiendo a una salud integral y a la necesidad de realizar una planificación conjunta para superar las distancias que venían sucediendo entre APS y los dispositivos de Salud Mental. Las autoridades hablaban de integrar "la isla" haciendo referencia a la DPSM con el resto de las dependencias ministeriales.

Conforme a los relatos obtenidos en las entrevistas realizadas se destacó que fueron muchas las resistencias en los equipos de APS al momento de abrir las puertas a estos nuevos equipos. Esas resistencias tenían orígenes diversos: o bien el hecho de no ser un recurso 
estable del centro de salud, o bien debido a que el ingreso no se había planificado en conjunto con los equipos de atención primaria, sin consultar las demandas y procesos de trabajos de los equipos, o bien porque la lógica matricial no se lograba comprender o, incluso, por cierto temor a que estos equipos externos se volvieran un mecanismo de control de las prácticas en salud mental de los equipos de salud.

A su vez, los equipos del dispositivo matricial relataban cierta desconexión entre los trabajadores y sus coordinaciones, un desconocimiento de los equipos de cada zona respecto a las prácticas y composición de los otros equipos; así como también se apreciaba un repetitivo relato de malestar hacia los equipos de APS por "no comprender la función del dispositivo", por "rechazar las situaciones de salud mental", porque "solo demandan a psiquiatras y a la medicación" o porque no quedaba clara la función de las psicólogas y los psicólogos".

$\mathrm{Y}$ así, entre encuentros y desencuentros del dispositivo de apoyo matricial y los centros de salud se fueron tejiendo los relatos de las personas entrevistadas para esta investigación. Esos relatos insisten en expresar preocupación por la escasa planificación conjunta entre el campo de la salud mental, los proyectos y planes que necesita para cumplimentar la Ley y los diferentes niveles de atención en salud que parecieran desentenderse de la elaboración estratégica integral que se requiere.

\section{Acerca del Dispositivo matricial}

Hasta el momento de la investigación, este el dispositivo estaba conformado por cinco equipos compuestos, cada uno por psicólogas/os, psiquiatras, enfermeras/os, talleristas y operadoras/es comunitarios; distribuidos en cinco distritos de la ciudad con la tarea de abordar situaciones de alta complejidad subjetiva, derivadas de los centros de salud provin- ciales de sus respectivas zonas.

Se observó que los equipos interdisciplinarios mostraban diversa constitución tanto en la cantidad de integrantes como en las disciplinas y funciones de cada uno; sin justificaciones, ni información respecto de los criterios con que se armó y definió cada equipo, en términos de características institucionales, mayores demandas de salud de las poblaciones o criterios de planificación en salud mental. Las variables cantidad de trabajadores y funciones dieron como resultado equipos heterogéneos, tanto en su conformación como en sus modalidades de trabajo ${ }^{3}$.

Las coordinaciones han variado reiteradas veces sin lograr establecer una dinámica con los equipos. Tal como fue reiterado en todas las entrevistas individuales realizadas a los integrantes del dispositivo: "es una coordinación que está más en un lugar de paridad y muchas veces, si uno no resuelve algo, nadie lo resuelve, no es posible agendar situaciones". A su vez, se destacaba la falta de acompañamiento de parte de directivos, la poca formación para trabajar de manera comunitaria y hacerles lugar a las prácticas matriciales, la insistente pregunta por las funciones y tareas de psicología, operadores comunitarios y talleristas que no quedaban claras; o el malestar por la sola demanda de los centros de salud a las y los psiquiatras del dispositivo.

Estas tensiones no encontraron algún espacio de puesta en común ya que desde la conformación del dispositivo las propuestas de encuentro de parte de la coordinación han

\footnotetext{
${ }^{3}$ Es importante destacar que en la actualidad esta característica se ha profundizado, conforme lo relevado en contactos con trabajadoras y trabajadores del dispositivo. Se brindó de ejemplo la desproporción entre las cargas horarias y la cantidad de efectores entre dos equipos del mismo dispositivo donde un equipo cuenta con una psicóloga y un psiquiatra para siete centros de salud de referencia mientras otro equipo de otra zona tiene dos psicólogas y tres psiquiatras para cinco centros de salud.
} 
sido muy pocas y las convocatorias realizadas por algunas trabajadoras y trabajadores por no lograr contar con la participación e interés de todos los equipos, fallidas.

En la entrevista realizada a una autoridad de la DPSM se observa que no se halla establecida una dinámica de reuniones o articulación entre los diferentes dispositivos o coordinadores:

\section{La verdad que lo veniamos haciendo, pero ahora hace un tiempo atrás no lo venimos ha- ciendo, pero la idea que todos los que estamos en este otro nivel nos reunamos y podamos discutir lo que está pasando. De todas ma- neras, yo me reúno constantemente con cada uno. (...) Al estar yo acá tengo contacto con todos ellos; y eso bizo poder tener una visión global de lo que está sucediendo en todos los dispositivos. (entrevista realizada como parte de la investigación ${ }^{4}$.}

Estas expresiones podrían dar cuenta de una lógica de trabajo que no prevé espacios para el diseño o planificación de las políticas según análisis de datos o relevamiento de problemáticas. Pareciera no contar con alguna formalidad, sino, más bien, con la lógica de lo espontáneo, donde todo recae sobre la figura de estas autoridades.

Resulta relevante destacar que ningún entrevistado o entrevistada nombra al dispositivo de la misma manera y que si bien se elaboró un documento con los lineamientos básicos del dispositivo no ha aparecido en los relatos como fundamento para las prácticas. Diferentes versiones del documento, pocas reuniones para su revisión o el enojo de parte de las psicólogas y psicólogos de los centros de salud por no haber sido consultados en

\footnotetext{
${ }^{4}$ Excepto que se aclare lo contrario, todos los fragmentos de este artículo en cursiva y con una letra menor corresponden a fragmentos de entrevistas realizadas a los fines de la investigación que aquí se presenta.
}

la elaboración y diseño de estos dispositivos fueron algunas respuestas que se pudieron estimar.

En el documento elaborado en 2012 se encuentra una definición precisa del dispositivo, que encuadra su metodología de trabajo dentro de las políticas sustitutivas al manicomio. Asimismo, es suficientemente clara la delimitación de las tareas de cada integrante del equipo y se destaca el acompañamiento a los equipos de centros de salud en el enlace con otras instituciones, la construcción de diagnósticos clínicos, o comunitarios, consultorios conjuntos, visitas domiciliarias, coordinación de espacios grupales y comunitarios, construcción de espacios de retrabajo clínico, y promoción de la salud como las más representativas de una práctica matricial. También pueden observarse las responsabilidades de la coordinación, cuyas tareas primordiales son la operativización de reuniones de retrabajo y acompañamiento con los equipos, la producción de intercambios y articulaciones con otras instituciones, la generación de espacios de encuentro para la formación de los trabajadores.

Conforme al análisis de diferentes versiones del documento constitucional del dispositivo, se puede observar un corrimiento y posterior borradura de lo matricial como forma de identificar las prácticas del dispositivo. En la entrevista realizada, el personal directivo de salud mental argumentó que se fue sacando el concepto de lo matricial porque "era muy dificil de explicar y generaba confusión entre los trabajadores". Sin embargo, en la mayoría de las entrevistas apareció el concepto de apoyo matricial como definición primordial que hacen de estos equipos y como práctica innovadora por la cual requieren formación específica.

Es llamativa la distancia -que se reitera en todas las entrevistas- entre la formalidad de la letra de los documentos normativos del dispositivo y las valoraciones que realizan sus trabajadoras y trabajadores respecto a la ausencia de lineamientos, direcciones claras 
y acompañamientos de políticas de gestión para facilitar el acceso a la salud mental territorial.

\section{Ensayos hacia una práctica de apoyo matricial}

En torno a las prácticas del Dispositivo matricial en el ámbito de la APS, se observa que con el tiempo cada equipo fue construyendo en su centro de salud alguna modalidad particular de trabajo, con diferentes dinámicas, prioridades, construcciones y dificultades.

Una tensión desplegada en torno al apoyo matricial aparece en la confrontación entre las prácticas de atención directa y las prácticas sin atención directa. Las primeras son pensadas como la atención de especialistas (por ejemplo, la asistencia de psiquiatría, recurso que no tienen los equipos de APS) mientras que las prácticas sin atención directa a la población están más bien identificadas con las reuniones por casos y elaboración de estrategias conjuntas a los equipos de referencia. Ambas modalidades aparecen tensionadas en las definiciones respecto al apoyo matricial que han elaborado las entrevistadas y los entrevistados, como si se tratara de una u otra y no pudieran articularse. Sin embargo, esta tensión se expresa y se pone en acto en las prácticas matriciales, incluso conviven dentro de un mismo equipo.

Se podría observar en estas dinámicas organizacionales cómo aparecen en un mismo equipo dos lógicas paralelas. Por un lado, los circuitos quincenales de rotación que tienen las y los psiquiatras, destinados a asistir las listas de pacientes de cada centro de salud; $y$, por el otro, la dinámica del resto del equipo destinada al armado de reuniones interdisciplinarias para la elaboración de estrategias clínicas de situaciones de complejidad subjetiva, la recepción de un pedido de intervención, aunque esté dirigido a psiquiatría- o, en otros casos, para sostener espacios semanales de retrabajo clínico ${ }^{5}$ y espacios comunitarios. Estas tareas parecen no lograr contar con la presencia de psiquiatras por dedicar la mayoría de sus horas al trabajo en consultorio.

Otra tensión se encontró respecto a las prácticas de las y los psiquiatras en APS. Si va pocas horas, si tiene demasiados centros de salud a su cargo, si es accesible o difícil de lograr una reunioncita -expresión dicha entre risas en referencia al anhelo de un tiempo que costaba hacerse, cada vez que se cruzaban con el psiquiatra de su zona.

En torno a esto un personal directivo expresó:

Cada centro de salud quiere tener un psiquiatra, pero no es lo que la DPSM piensa lo que se debe hacer, porque estamos tratando de readecuar los monovalentes, estamos en una lógica de trabajo donde la persona esté lo más cerca de su casa posible, que pueda ser incluido dentro de la sociedad, dentro de la comunidad. Si ponemos un psiquiatra como un servicio de salud mental dentro de un centro de salud vamos con una lógica que va en contra pelo de lo que nosotros pensamos.

Sin embargo, la mirada de las y los psiquiatras del dispositivo parece disentir con esta lectura:

En el caso general de los psiquiatras, somos los que menos horas tenemos. Entonces, yo siento que esto me termina confinando al consultorio, y que no tengo otro tiempo para hacer esto que digo necesario, para poder trabajar de otro modo, que implicaría poder reunirme más, trabajar con los equipos, trabajar con otras instituciones. O sea, más allá de lo

\footnotetext{
${ }^{5}$ Las entrevistadas y los entrevistados utilizan esta expresión para nombrar las reuniones con los equipos de referencia de usuarias y usuarios de la salud en las cuales se piensan y revisan los proyectos terapéuticos.
} 
asistencial, de consultorio directo al paciente, tener la posibilidad de trabajar en la estrategia clinica y poder incidir, también, sobre esas estrategias, que, bueno, es difícil por la carga horaria. Muchas veces la respuesta que dan nuestros directivos en relación a la hora de los psiquiatras tiene que ver con que se piensa que más horas del psiquiatra es hegemonizar una práctica o manicomializar y para mi es justamente todo lo contrario. Menos horas implica que me quede totalmente por momentos automatizada a la práctica de consultorio y no tenga otro tiempo para pensar.

Esta tensión expresada en la última cita se reitera en los trabajadores de los centros de salud quienes comentan que priorizan la demanda de la especialidad de psiquiatría a la hora de convocar al dispositivo porque es un saber específico que no tienen las médicas y los médicos generalistas, justificándose en el desconocimiento de otras disciplinas para abordar las problemáticas subjetivas o desde la incomodidad institucional que produce el tener que derivar a psiquiatras de los hospitales, ya sea por la poca disponibilidad de turnos o la insuficiente articulación y seguimiento clínico de pacientes entre los dos niveles de atención.

Así, cada 15 días, los centros de salud provinciales cuentan con una imagen que se reitera: salas de espera con "los pacientes de salud mental" o pacientes cuya "propiedad" -como si se tratara con objetos o cosas- está asignada al apellido del o la psiquiatra de referencia, para su control y tratamiento.

El debate circula en torno a la pregunta: “¿De quién es el paciente?”. Pacientes de psiquiatría o pacientes de salud mental aparecen en las nominaciones de los equipos de APS. $\mathrm{El}$ intento de armarle un equipo interdisciplinario de referencia a cada persona que consulta por salud mental aparece como uno de los desafíos más nombrados por las y los integrantes del dispositivo.
Algunas conceptualizaciones teóricas pueden ser esclarecedoras en este momento. El apoyo matricial es pensando como espacio para la construcción de un proyecto terapéutico integrado entre equipo de referencia y apoyadores. Puede desarrollarse en tres planos fundamentales:

a) Atenciones e intervenciones conjuntas entre el especialista matricial y algunos profesionales del equipo de referencia; b) en situaciones que exijan atención específica al núcleo de saber del apoyador, este puede programar para sí aún una serie de atenciones o de intervenciones especializadas, manteniendo contacto con el equipo de referencia, que no se descomprometería con el caso, al contrario, buscaría redefinir un patrón de seguimiento complementario y compatible al cuidado ofertado por el apoyador directamente al paciente, o a la familia o a la comunidad; c) es posible aunque el apoyo se restrinja al cambio de conocimiento y de orientaciones entre equipo y apoyador; diálogo sobre alteraciones en la evaluación del caso y aún reorientación de conductas antes adoptadas, permaneciendo, pero, el caso bajo cuidado del equipo de referencia (Campos, Domitti, 2006, p.1549)

Esa dinámica, expresada en encuentros periódicos, se presta tanto para ordenar la relación entre los niveles jerárquicos del sistema cuanto para facilitar la comunicación e integración de equipos de salud de la familia y especialistas, o aún entre distintas especialidades y profesiones de salud que trabajen en un mismo servicio, hospital o centro de atención.

En la investigación se analizó que ante una situación de complejidad subjetiva el dispositivo podía responder desde dos lógicas observadas. Una identificada más bien a los mode- 
los clásicos de atención biomédica; otra que busca respuestas desde la clínica de la subjetividad, apuesta a estrategias interdisciplinarias, intersectoriales y comunitarias de abordajes para incidir en los arreglos institucionales a fin de alojar las problemáticas en salud mental.

La primera es una lógica que repite ciertos circuitos médico-hegemónicos de atención, ofreciendo evaluación psiquiátrica ante la consulta del equipo de APS. Aquí las psicólogas y los psicólogos del dispositivo y operadoras y operadores aparecen como acompañantes o auxiliares de psiquiatría: escuchan la demanda, facilitan la llegada y accesibilidad de medicación, realizan consultorio conjunto con psiquiatras bajo el argumento de estar al tanto de los pacientes que consultan por psiquiatría para que no sean solo los pacientes de psiquiatría. Esta práctica es leída por los equipos de APS de modo crítico, por la generalización de esta modalidad, sin miramientos a las singularidades. Explican que muchas veces las usuarias y los usuarios ya cuentan con un espacio de psicología y son nuevamente sometidos a una escucha psi, sin ser parte de la estrategia singular, generando confusión de los roles y tareas de cada actor. Una psicóloga de un centro de salud agrega:

\section{A mi gusto termina siendo una práctica com- pletamente manicomial, porque en definitiva lo único que importa que esté es el psiquiatra y lo único que el equipo pide si o si es el psi- quiatra... Los otros parecieran ser como una especie de mampostería de adorno que están o no están y no hay mucha diferencia}

La permanente derivación a psiquiatría, las largas listas de pacientes, la sola demanda al psicofármaco como modo de contener y abordar las problemáticas de salud mental y las respuestas desde los dispositivos clásico como el consultorio y la dupla psiquiatría-psicología sigue operando hegemónicamente en los servicios de salud. APS no parece estar exenta de eso.
En los equipos con estos modelos de atención pareciera identificarse la práctica de apoyo matricial al tratamiento de pacientes cuya complejidad subjetiva requiere la indicación de psicofármacos. El equipo realiza la evaluación y seguimiento de estos pacientes. Pareciera a su vez abordarse la salud mental centrando la atención en la supresión sintomática del consultante y el tratamiento individual.

Lo matricial aparece aquí como estar al llamado de los equipos de los centros de salud cuando crean necesaria la intervención del equipo especializado en salud mental; para lo cual crearán alguna dinámica de trabajo específica según lo requiera el caso. Se describen, por ejemplo, visitas a los hogares, evaluaciones en consultorio, reuniones con los equipos o articulación con otras instituciones. La pregunta y desconocimiento en torno al lugar y función de psicología y operadores comunitarios fue recurrente.

Se contrapone a este modelo otras formas de abordajes que fueron relevadas en la investigación, donde se buscan otras formas de respuesta que contemplen la lectura social y comunitaria de las problemáticas en salud mental.

Algunos equipos del dispositivo matricial construyeron otras dinámicas de trabajo junto a los equipos de APS corriendo del centro de la escena a la figura del o la psiquiatra e instalando en cada centro de salud algún dispositivo de abordaje según las características poblacionales y las necesidades evaluadas en conjunto por las instituciones de referencia. La presencia de estos equipos en los centros de salud no es al llamado sino periódica.

Las reuniones regulares interdisciplinarias de situaciones complejas aparecen como la condición de trabajo en cada centro de salud a los que acude el dispositivo matricial, proponiendo estrategias concretas para esa situación específica. Según la propia descripción de los equipos, sus tareas pueden abarcar 
desde efectuar reuniones con los equipos de los centros de salud, promover y coordinar reuniones con otras instituciones u organismos, acompañar la gestión de acompañamientos terapéuticos y retrabajar, junto a cada acompañante, el material clínico; promover y participar de espacios grupales y artísticos; realizar visitas, llamados y asistencia en consultorio en situaciones donde se evalúe necesario sumar este recurso. Psiquiatría forma parte de estas reuniones interdisciplinarias acompañando con formación y escucha a otras disciplinas.

Según se relata en una entrevista, el sostenimiento de estos espacios nombrados como reuniones de situaciones

permite no solo el desarrollo de tareas para
avanzar con la resolución de alguna de las
situaciones planteadas, sino que, también,
permite una oportunidad para acompañar a
los trabajadores a reflexionar sobre sus modos
de respuestas, sus implicancias o resistencias
con determinados pacientes, así como también
permite realizar preguntas para elaborar
respuestas colectivas y comunitarias ante las
problemáticas más recurrentes

Las reuniones de seguimiento de casos y revisión de estrategias con cierta frecuencia es una condición necesaria a la hora de abordar un caso al modo matricial y es una manera de continuar promoviendo la referencia y responsabilidad compartidas.

La asistencia directa en consultorio de las psicólogas y los psicólogos o de psiquiatría se decide en estas reuniones destinadas a pensar las estrategias territoriales y proyectos clínicos. Sin embargo, el abordaje no se reduce a la atención en consultorio, sino que se despliegan espacios poniendo a disposición los recursos artísticos y populares de las y los talleristas y operadoras y operadores de los equipos e integrando a la población con las trabajadoras y los trabajadores de los centros de salud.
Ofrecer espacios grupales de abordaje y en la comunidad ante una demanda de atención individual suele ser una modalidad recurrente de estos equipos. Talleres de murgas en escuelas o en las puertas de un centro de salud, talleres de nutrición y comida saludable, caminatas, yoga, ensamble de tambores, espacio de adolescentes en las plazas o fútbol son algunas de las propuestas comunitarias llevadas adelante por el dispositivo. Para ello, se apuntalan de las herramientas lúdicas de las y los talleristas y convocando a médicas, médicos, psicólogas, psicólogos, trabajadoras y trabajadores sociales, administrativas, administrativos y enfermeras o enfermeros del centro de salud para el retrabajo clínico y sostenimiento. El hecho de ser propuestas diseñadas y sostenidas sobre todo por los equipos matriciales genera cierta continuidad en las prácticas grupales que los mismos equipos de APS admiten no lograr debido a la sobrecarga de demanda de atención.

$\mathrm{Si}$ bien en algunos equipos las intervenciones se agotan en el clásico dispositivo de consultorio mientras que en otros se elaboran respuestas que incluyen la comunidad; el apoyo matricial está siempre como horizonte de sus prácticas. La promoción de reuniones con los equipos de referencia para transmisión de las situaciones complejas que se quieren derivar y el modelo organizacional común en el cual los integrantes del dispositivo no son quienes realizan la primera escucha o demanda de atención de la población; permite comenzar el abordaje por el retrabajo clínico e intercambio junto a estos equipos de atención primaria. Este tiempo para pensar, para ordenar la complejidad, apareció en las entrevistas como una forma de práctica que habilita otras clínicas y como ocasión para la formación.

En la mayoría de las entrevistas, este tiempo para pensar ha aparecido como aquello que falta en el hacer cotidiano, un hacer arrasado por la acción, el resolver desde lo inmediato, con poco espacio a alguna pausa que 
permita la revisión de ese hacer y la ideación de nuevas estrategias.

Quizás estos espacios para pensar sean representados en APS como un ideal difícil de alcanzar y promoverlos y sostenerlos se trate de una tarea de equipos que no estén en la asistencia directa, como forma de construcción de la clínica ampliada.

Estos espacios, además, se vuelven ricos como ocasión para discutir sobre modelos de organización de la demanda, reflexionar el tipo de respuesta y contacto con la población, realizar análisis situacionales de las problemáticas más emergentes para pensar estrategias institucionales y comunitarias como modo de abordarlas. Así, la tarea de estos dispositivos se convierta, tal vez, en una práctica tanto clínica, como institucional y comunitaria.

\section{Reflexiones finales. Lo matricial como hilo que enlaza}

La pregunta acerca de lo matricial, sus modalidades de implementación y lineamientos ha ido a apareciendo a lo largo de toda la investigación, como una llave que permitía pensar en esas otras prácticas de cuidado necesarias de desplegar en el campo de la salud mental.

Si bien se relevaron en los equipos del dispositivo diferentes experiencias de abordaje -algunas más ligadas a los modelos clásicos y otras en la búsqueda de nuevas prácticas-, lo matricial aparecía con insistencia en cada relato como ideal de una práctica a lograr, como propuesta que invitaba a transformar los modos de alojamiento de la locura y el sufrimiento subjetivo en los espacios públicos de salud.

Es posible analizar que estos equipos, con la pretensión de resistir las prácticas de encierro y manicomialización de la locura, buscasen la creación de diferentes espacios para problematizar las situaciones de mayor complejidad subjetiva presentadas como de- mandas de atención a los equipos de APS. Buscaban alojar de manera interdisciplinaria e intersectorial, a la vez que se proponían diversas experiencias grupales poniendo el arte o herramientas del saber popular como vehículos para enlazar-nos a la población, visibilizar sus problemáticas y la creación conjunta de estrategias colectivas para su elaboración. Estos equipos, tejiendo lazos, buscaban oficiar de enlace entre las diferentes instituciones, organizaciones y dimensiones que hacen al campo de la salud mental.

Como se observó, cada centro de salud tenía perfiles diferenciales claramente identificables en cuanto a los procesos de atención, a las modalidades de ordenamiento interno, a la accesibilidad de la población, a los arreglos organizacionales, a los modelos para responder a la demanda o en la relación con la comunidad.

La heterogeneidad en los centros de salud invita quizás, a que los apoyos matriciales adquieran características diferenciales en cada uno y a que las propuestas o modalidades de trabajo de estos dispositivos varíen según los diferentes procesos de trabajo que construye cada institución. Se hace pertinente preguntarse entonces, si no es esto un aspecto a tener en cuenta al momento de pesar el diseño y la planificación de estos equipos matriciales.

Sin embargo, en cada entrevista apareció con claridad la ausencia de datos epidemiológicos respecto a las poblaciones con quienes trabajan estos dispositivos, un desconocimiento de las prácticas e intervenciones que se realizan y una distribución arbitraria y desorganizada de los recursos sanitarios; elementos que nos llevan a preguntarnos cómo se piensa la planificación en salud mental en la provincia de Santa Fe cuando no se cuenta con datos concretos de las poblaciones y sus problemáticas más emergentes, ni datos institucionales que permitan realizar una lectura en torno a las modalidades de atención de la demanda, los aciertos, dificultades y desafíos 
a superar.

Rosana Onocko (2007) habla de "la planificación en el laberinto”, metáfora más que pertinente para pensar las dificultades que atraviesa la planificación en salud cuando sus directrices son construidas al margen de las lecturas poblacionales y de las construcciones que realizan las trabajadoras y los trabajadores. Las gestiones parecen perderse en los requisitos burocráticos, en la tarea de ser "el guardián del minotauro" (p.135) es decir, que lejos de innovar, se dedican a cuidar ciertas estructuras cristalizadas, tratándose de planificaciones de escritorios y sin estudios específicos de las problemáticas que necesitan transformar.

El trabajo de relevamiento de las problemáticas en salud mental más emergentes, las resoluciones que se le solicitan tanto al sistema público de salud como a otros sectores ministeriales, la creación de estrategias atentas a dar respuestas a los datos relevados y la asignación de presupuestos concretos para el diseño de una red sustitutiva parecieran ser horizontes claros para las trabajadoras y los trabajadores de la salud pero que no logran tomar fuerza en las agendas políticas de Estado.

En torno a estas reflexiones, resulta apropiado citar la investigación realizada en Argentina por el CELS (2015) donde se problematizan las deficientes políticas implementadas para generar un proceso de sustitución manicomial, ubicando que gran parte del presupuesto asignado a las políticas de salud mental siguen centradas en las instituciones psiquiátricas y en los psicofármacos.

En todas las jurisdicciones siguen siendo escasos los dispositivos de salud mental centrados en la comunidad (casas de convivencia, casas de medio camino, hospitales de día, hospital de noche y centros de día) y con carácter móvil para la atención en domicilio. Hasta ahora los dispositivos existentes son básicamente los mismos que existían antes de la LNSM, y sólo algunos han recibido refuerzos presupuestarios y en su planta de recursos humanos. Un dato llamativo es que la mayoría de esos dispositivos siguen dependiendo financiera e institucionalmente de los hospitales psiquiátricos ( $\mathrm{p} 46$ )

Las políticas en nuestra provincia no parecen estar ajenas a los estudios arrojados por esta investigación. El plan hacia lo sustitutivo no aparece. Algunas experiencias aisladas, desarticuladas entre sí, con bajo presupuesto y con escasa planificación intentan producir alternativas, pero, mientras tanto, lo manicomial resiste y la vulneración de derechos que este modelo implica, también.

Pensando en el sistema de salud, Gastón W. de Sousa Campos (2001) propone dispositivos que produzcan otra cultura y otras líneas de subjetivación, en detrimento de las estructuras inductoras de corporativismo $\mathrm{y}$ alienación, donde se piensa al usuario de salud como un consumidor o un sujeto pasible de soportar el intervencionismo de la medicina hegemónica. Dispositivo se entiende aquí como máquina para hacer ver y hacer hablar (Deleuze,1990), como un "encuadre de trabajo" (Minnicelli, 2008), un artificio; una herramienta en permanente construcción, crítica, reflexiva y atenta a las circunstancias o contextos clínicos, políticos e institucionales en donde se desarrolla.

En este sentido, el apoyo matricial es una propuesta que interpela los modos clásicos de asistencia, centrados en la mirada biomédica, o de consultorio cerrado; que recupera voces y experiencias comunitarias, que interpela modelos de atención y propone prácticas instituyentes, que devuelve subjetividad a la clínica del cuidado, respetando diversidades y promoviendo autonomía. Se pretende que auxilie al equipo de referencia en la formulación, reformulación, ejecución de un proyecto te- 
rapéutico singular para un sujeto individual o colectivo que necesita una intervención en salud. A su vez se espera que el equipo de apoyo matricial favorezca la creación de espacios de discusión sobre las relaciones institucionales, apuntando a crear o transformar ciertas dinámicas a la hora de abordar el malestar social y subjetivo.

Podría reiterarse entonces, que los dispositivos matriciales investigados son capaces de operar tanto en el plano de lo clínico como de lo institucional y comunitario, oficiando como hilo que se despliega entre el afuera y el adentro de las instituciones de salud, enlazando y tejiendo redes entre las diferentes dimensiones que hace al campo de la salud mental. Permiten, así, dar pasos hacia la creación de redes sustitutivas para lograr el cierre de los manicomios y superar sus lógicas.

\section{Referencias}

- Amarante, P. (2006). Locos por la vida Trayectoria de la reforma psiquiátrica en Brasil. Buenos Aires: Asociación Madres de Plaza de Mayo.

- Ballarin, M.; Blanes, L; Ferigato, Sh. (2012). Apoio matricial: um estudo sobre a perspectiva de profissionais da saúde mental. En Interface- Comunicação, Saúde, Educação, vol16, N42. Pp. 767-778

- Bleichmar, S. (2001). "Efectos de un pensamiento crítico en la práctica y la teoría" En Revista La Oreja, psicoanálisis y pensamiento crítico. Facultad de Psicología. Universidad Nacional de Rosario. Disponible en enhttps://es.scribd.com/ document/67285283/Efectos-de-unpensamiento-critico-en-la-practica-yla-teoria

- Campos, G. (2001). Gestión en Salud. Buenos Aires: Lugar.

- (2002) Reflexões sobre a clínica ampliada em equipes de saúde da família. Disponible en https://www.trabalhosfeitos.com/ensaios $/$ Reflex $\%$ C3\%B5es-Sobre-a-Cl\%C3\%ADnica-Ampliada-Em/64732756. html

- (2007) "Reforma política e sanitária: a sustentabilidade do SUS em questão?". En Ciência \& Saúde Coletiva, 12(2). Pp.301306.

- y Domitti, AC. (2006). "Apoio matricial e equipe de referência: uma metodologia para gestão do trabalho interdisciplinar em saúde". En Cadernos Saúde Pública, Rio de Janeiro, 22(X). Pp. 1547-1555.

- Castro, C. y Oliveira, M. (2016). "Apoio Matricial no SUS Campinas: análise da consolidação de uma prática interprofissional na rede de saúde". En Ciencia \& saúde Colectiva, 21 (5). Pp.1625-1636.

- Centro de Estudios Legales y Sociales (2015). Informe anual: Cruzar el muro: desafíos y propuestas para la externación del manicomio. disponible en http://www.cels.org. ar/especiales/cruzarelmuro/

- DECLARACION DE ALMA-ATA (1978) Conferencia Internacional sobre Atención Primaria de Salud, Alma-Ata, URSS,

- Faraone, S. y Valero, A. (comps.) (2013). Dilemas en Salud Mental. Sustitución de las lógicas manicomiales. Buenos Aires: Madres de Plaza de Mayo.

- Galende, E. (2008). "Desmanicomialización institucional y subjetiva". En Psicoanálisis. vol. XXX-N²/3. Pp.395-427.

- Gerlero, S., Augsburger, A. C., Yanco, D., y Duarte, A. P. (2011). "Salud Mental y Atención Primaria, accesibilidad, integralidad y continuidad del cuidado en centros de salud". En Argent Salud Pública, vol 2. Pp 24-29

- Laurell, A. C. (2010). Revisando las politicas y discursos en salud en América Latina. Revista Medicina Social. Vol. 5, Numero 1. (www.medicinasocial.info)

- Merhy Ee, F. L. (2012). Contribuciones 
metodológicas para estudiar la producción del cuidado en salud: aprendizajes a partir de una investigación sobre barreras y acceso en salud mental. SALUD COLECTIVA, 8(1):25-34.

- Onocko, R (1999) La clínica ese método olvidado por la salud pública. (Mimeo)

- (2007) La planificación en el laberinto: un viaje bermenéutico. Buenos Aires: Lugar.

- Ministerio de salud de la nacion (2013). Plan nacional de salud mental. Disponible en http://www.msal.gov.ar/saludmental/ images/stories/info-equipos/pdf/201310-29_plannacional-salud-mental.pdf

- Silveira, E. (2012). "Práticas que integram a saúde mental á saúde pública: o apoio matricial e a interconsulta". En Ciencia \& saúde Colectiva, 17(9). Pp. 2377-2386.

- Stolkiner, A.; Comes, y; Garbus, P (2011). "Alcances y potencialidades de la Atención Primaria de la Salud en Argentina". En Ciencia \& saúde Colectiva, 16 (6). Pp.
2807-2816.

- y Ardila Gomez, S. (2012) "Conceptualizando la Salud Mental en las practicas: consideraciones desde el pensamiento de la medicina social/salud colectiva latinoamericanas". En Vertex Revista Argentina de Psiquiatría. vol XXIII\}. Pp. 57-67

- y Solitario, R. (2007) "Atención Primaria de Salud y Salud Mental: la articulación entre dos utopías." En Maceira, D. (comp). Atención Primaria en Salud-Enfoques interdisciplinarios. Pp. 121-146. Buenos Aires: Paidos.

- Testa, M. (2009). Pensar en salud. Buenos Aires: Lugar.

- (1985). "Atención Primaria o atención primitiva". En Cuadernos médicos-sociales, No34: Rosario. Pp. 7-21.

- Ulloa, F. (1995). Novela Clínica Psicoanalitica. Historial de una práctica. Buenos Aires: Paidós. 